\title{
Direct determination of astronomical distances and proper motions by interferometric parallax
}

\author{
P. Jain ${ }^{1}$ and J. P. Ralston ${ }^{2}$ \\ ${ }^{1}$ Physics Department, IIT, Kanpur 208016, India \\ e-mail: pkjain@iitk.ac.in \\ 2 Department of Physics and Astronomy, University of Kansas, Lawrence KS 66045, USA \\ e-mail: ralston@ku.edu
}

Received 14 September 2007 / Accepted 13 April 2008

\section{ABSTRACT}

\begin{abstract}
Aims. We discuss a new method for measuring the distances to astronomical objects and their transverse proper velocities. Methods. The phenomenon of interferometric parallax identifies a component of 2- and 4-point amplitude and the intensity correlations that can be observed at frequencies ranging from radio to optical. The calculation hinges on terms depending on the source to receiver separation that are conventionally neglected in the Van Cittert-Zernicke theorem.

Results. Order of magnitude estimates find that the baselines of Earth-bound VLBI systems might be capable of measuring 10-100 kpc distance scales. In either the optical or radio regime, space-borne detectors with fine baseline control might resolve source distances of Gigaparsec order. We discuss the possibility that an independent distance ladder based directly on experimental measurement might be constructed on multiple scales.
\end{abstract}

Key words. techniques: interferometric - astrometry - instrumentation: interferometers

\section{Introduction}

No problem in astronomy is more important than resolving the third dimension of source distances. Measurements of transverse proper velocities of distant objects has been equally elusive. Here we discuss a new and attractive method that is modelindependent and needs no special assumptions about source properties.

Finding distances by the traditional method of trigonometric parallax is a geometrical procedure requiring precise resolution of angular positions. The most ambitious distances measured with trigonometric parallax are currently of order kpc (Bradshaw et al. 1999; Hachisuka et al. 2006; Bartel 1988; Brisken et al. 2002; Campbell et al. 1996; Dodson et al. 2003; Honma et al. 2000; Sjouwerman et al. 2004; van Langevelde et al. 1999; Vlemmings et al. 2005). A future Space Interferometry Mission ${ }^{1}$ (SIM) aims to extend this range to $10 \mathrm{kpc}$. Methods to measure distances beyond trigonometric parallax do exist: Pulsars provide an independent approach via observation of the phase of their periodic signal (Gwinn et al. 1986a,b). Larger distances are measured by a method based on "standard candles", which relies on assumptions and models concerning the absolute luminosity of objects.

The new method developed here exploits a phenomenon we call interferometric parallax. The mechanism differs substantially from traditional parallax used for centuries. With interferometric parallax no precise measurement of object angular positions is needed. It is remarkable that the individual sources do not need to be resolved by the instruments. Moreover, a measurement of transverse source velocities relative to the observer are not inherently limited by the ability to track the source's

${ }^{1}$ SIM is reviewed in http://planetquest.jpl.nasa.gov/SIM/ sim_index.cfm angular position. While it may seem impossible to develop parallax between unresolved sources, it is a simple consequence of established physics used in a new way. Sources should be close to one another in angular position, by criteria listed below, but few restrictions exist otherwise. Finding sources that meet the necessary conditions does not appear to be a barrier. Pulsars or radio maser sources in our galaxy can be compared to a distant galaxy, whose distance at apparent infinity drops out of the measurement. A galaxy, in turn, can be measured relative to AGN at apparent infinity. A cosmologically distant source at redshift $z \sim 1$ might be compared to one with $z \sim 2.5$, and so on. It seems possible to build up an independent "distance ladder" using the method without needing to refer to other methods of distance determination.

In Sect. 2 we extend the Van Cittert-Zernicke (VCZ) theorem, which describes the correlations between receiver pairs that monitor uncorrelated sources. The VCZ theorem has long been developed in plane wave approximation that might seem exact for very distant sources (Mandel \& Wolf 1995; Scully \& Zubairy 1997; Gupta \& Dwarakanath 1999). Actually astronomical wave fronts are slightly curved. The effects of curvature can be observed by monitoring the interference of both amplitude and intensity correlations. We will show that the translational dependence of receiver correlations leads directly to determination of the distance to a source.

Since everything about wave optics is established physics, several "working parts" of our analysis can be found in one context or other in the literature. The novelty of our approach lies in how the parts are assembled. In Sect. 2 we identify the curvature corrections to the VCZ theorem, given in Eqs. (3) and (6) below. The Taylor expansions of the phase shifts necessary for this task have been done many times before. They are closely related to Fresnel-zone corrections. Curvature corrections also appear 
in the precision calculations of VLBI time delays in the solar system (Kopeikin \& Schäfer 1999) and gravitationally-induced curvature of wave fronts (Samuel 2004). A prestigious review article on radio astronometry and geodesy (Sovers et al. 1998) also discuss the order of magnitude of curvature corrections. The curvature corrections contain everything needed for parallax measurements. Yet those working in optical and radio astronomy continue to rely on making images in plane-wave approximation. Upon initiating the image-making process, trigonometric parallax is then limited by image resolution (Kurayama et al. 2005; Dodson et al. 2003; Vlemmings et al. 2003, 2005). Image making in radio astronomy is a data-intensive process of combining many "slices" from correlated detector pairs crossing an object multiple times and normally accumulated over a long running period (Thompson et al. 2001). A great deal of redundancy, signal processing and heuristic cross-checks go into making even the simplest images.

There is a gap between accounting for wave-curvature effects and parallax effects. A likely explanation for neglecting curvature effects is the order of magnitude estimates that picosecond time resolution cannot resolve distances larger than about $10 \mathrm{pc}$ (Sovers et al. 1998). It turns out that a simple change of variables revises the order of magnitude. The cumulative run-out of phase shifts is not taken into account by simple dimensional analysis, so that wave curvature can sense distances of unlimited extent. As we will show it is also completely unnecessary to make images. The time-intensive procedures of scanning in the " $u-v$ " plane of wave numbers can be eliminated in order to focus on much more simple and basic measurements of the particular phase shifts that distance measurements pinpoint.

The possibility to use intensity correlations for distance measurements is also fascinating. Two-point and multi-point intensity correlations at optical frequencies can be measured by instruments that simply count photons. Since the wavelengths are shorter than radio the ability to measure fine effects is correspondingly increased. Intensity correlations were used decades ago to measure stellar diameters (Hanbury-Brown \& Twiss 1954, 1956), but the technique has gone out of fashion. We find special features of two and four-point correlations that suggest direct determination of distances can be automated using "data against data" to remove unwanted effects.

The wavelength regime for "best application" depends on many technical details, and it would be premature for us to engage those questions in this paper. Rather than unwisely restrict the applications, we concentrate on developing general features without favoring any particular realization. We also choose not to discuss general relativity and its framework of interpretation. It is a straightforward but model dependent task to explore how the local wavefront curvatures are related to cosmological features such as the Hubble expansion. We nevertheless give a brief exposition of the most exciting prospects for contributing to the current debate over cosmological distance determination, which seems to us possible using existing technology.

Section 2 starts with a simple example highlighting distance determination with pairs of detectors too crude to possibly form any images. This is followed by our extension of the VCZ theorem. After developing the mathematics a simple physical interpretation is given in Sect. 2.3. There are naturally complications, of which the main problem is controlling the fluctuations of a large, leading order phase. A new method for canceling this phase using data against data is given in Sect. 2.5. Because so much about the topic is conceptual, it would be ill-advised to get involved with technological or instrumental questions, which also lie beyond our expertise. We discuss conceptual applications in Sect. 3. Section 4 gives a brief summary and some conclusions. An Appendix discusses finite source size effects, and a strategy of optimal alignment.

\section{Interferometric parallax}

To begin our development we describe a conceptual example. Consider a primitive array consisting of two to four dipole radio receivers separated by typical distances $\Delta x$. Direct a singlechannel time-dependent signal of wavelength $\lambda$ from a distant source into each instrument. A relative phase $\phi$ can be extracted between each receiver pair. One pair suffices for discussion, while there are interesting advantages to two or more pairs. In no event is the information from one phase sufficient to create any kind of image, much less resolve the sources. Unlike the situation in which images are synthesized by repeated passes through many different relative orientations, the entire information will be limited to a few phase shifts, which however (by concentrating all effort on them) might be very well resolved. Now translate the array by a vector $\Delta \boldsymbol{X}$, and measure the phase shifts again. Subtract two phase shifts to get a difference-of differences $\Delta \phi$. Then to order of magnitude the distance to the nearest source is

$r \sim \frac{\Delta X \Delta x}{\Delta \phi \lambda}$

This summarizes the non-obvious workings of our new approach to measuring astronomical distances.

We mentioned earlier that phase shifts from wave curvature have been developed before. Several works, including Sovers et al. (1998) estimate the effects as follows. The lowest nontrivial effects of the curvature correction must go like $1 / r$. Explicit evaluation of the Taylor expansion identifies the curvature terms that might be resolved with 16-digit computer accuracy. The curvature time delay $\Delta t$ for receivers separated by $\Delta x$ is then of order $\Delta t \sim \Delta x^{2} /(r c)$. With terrestrial receivers separated by $\Delta x \lesssim 10^{4} \mathrm{~km}$, and resolving $\Delta t \sim$ picosec the effects of curvature are ignorable for $r \gtrsim 10$ pc (Sovers et al. 1998). The estimate is technically correct as written for receivers at fixed locations. Yet it overlooks the translational dependence, which represents a cumulative run-out of the curvature-induced phase shift. The translational dependence appears explicitly in Eq. (1) where $\Delta X \Delta x$ replaces $\Delta x^{2}$ in estimating the cumulative effect. One astronomical unit of $\Delta \boldsymbol{X}$ is about 22000 Earth radii, so that neglecting the cumulative run-out is not a small effect. Moreover, the appearance of 1 picosec, a radio gold-standard, tends to obscure the much more rapid phase shifts of wave curvature at optical frequencies.

It is somewhat subtle, and long overlooked, that the Van Cittert-Zernicke theorem itself does not predict any parallax at all. The theorem makes asymptotic distance approximations in the first step, which instructs the mathematics that all sources are at infinity. It is left to the ingenuity of the astronomer to ignore the literal theorem and interpret a shift in an image position as due to parallax. In reality the asymptotic plane wave approximation is inexact and wave curvature is responsible for all renditions of "parallax", whether measured by images or phase shifts. Once saddled with an inconsistent theorem and rules for imagemaking, the traditional approach cannot proceed without images of superb quality, whereby the determination of distances is entirely dependent on the resolution and overall image quality subject to many degrading factors.

Our first task is to extend the derivation of the Van Cittert-Zernicke theorem with a Taylor series expansion. 


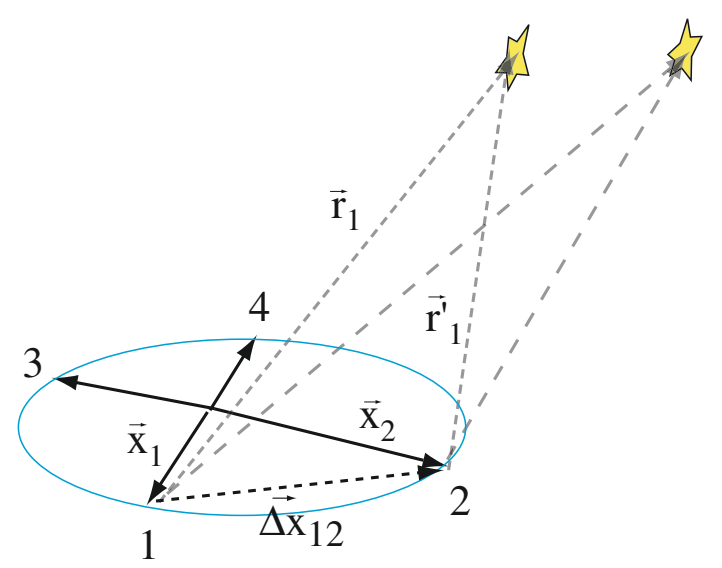

Fig. 1. Coordinate conventions of receivers and sources. The curvature phase depends on the net translation $\Delta \boldsymbol{X}_{12}$ of a receiver pair relative to sources. The figure shows an advantageous configuration of 4 receivers as discussed in the text.

We identify the essential degrees of freedom for distance determination which involves separating out the translational dependence. That simple step makes our first point of departure. Our second point of departure finds that there is no need for any images, nor image imperfections, nor the long running time needed to develop high quality images. This frees the astronomer to determine the particular phases that are relevant, without the overload of determining numerous pieces of information not directly related to the task.

\subsection{Extending the VCZ theorem}

We will present the material in self-contained fashion starting at the most basic level. Some care in defining coordinates and symbols is needed.

Let $\boldsymbol{X}_{0}$ be a local coordinate origin in the vicinity of the detectors. Consider for discussion two sources $S, S^{\prime}$ at positions $\boldsymbol{r}, \boldsymbol{r}^{\prime}$ relative to the local origin (Fig. 1). We assume these to be point sources. Finite size effects are discussed in the Appendix. We use primed symbols consistently, with $\boldsymbol{r}=\hat{\boldsymbol{r}} r$, $\boldsymbol{r}^{\prime}=\hat{\boldsymbol{r}}^{\prime} \boldsymbol{r}^{\prime}$ defining the source distances $r, r^{\prime}$ and unit vectors $\hat{\boldsymbol{r}}, \hat{\boldsymbol{r}^{\prime}}$ relative to $\boldsymbol{X}_{0}$. Let $\boldsymbol{x}_{J}$ be the position vector of the $J$ th detector. Relative to the detectors the sources are located at

$\boldsymbol{r}_{J}=\boldsymbol{r}-\boldsymbol{x}_{J}$

$r_{J}^{\prime}=r^{\prime}-x_{J}$.

The distance $r_{J}$ from the $J$ th detector to the source is

$r_{J}=r-\hat{\boldsymbol{r}} \cdot \boldsymbol{x}_{J}+\frac{1}{2 r} \sum_{i, j=1}^{3} x_{J}^{i} \delta_{\mathrm{T}}^{i j}(r) x_{J}^{j}+O\left(1 / r^{2}\right)$,

where $\delta_{\mathrm{T}}^{i j}(r)=\delta^{i j}-\hat{r}^{i} \hat{r}^{j}$. Upper indices denote vector components. Observe that $\delta_{\mathrm{T}}^{i j}(r)$ is a projector that removes the components of vectors parallel to $\hat{\boldsymbol{r}}$. Vectors multiplied by $\delta_{\mathrm{T}}^{i j}(r)$ effectively lie in the tangent plane of the sky.

Each source emits a given polarization of electric field $E_{\mathrm{S}}$, $E_{\mathrm{S}}^{\prime}$, with wave number $k$ and intensities $I_{\mathrm{S}}=\left\langle E_{\mathrm{S}} E_{\mathrm{S}}^{*}\right\rangle, I_{\mathrm{S}}^{\prime}=$ $\left\langle E_{S}^{\prime} E_{S}^{\prime *}\right\rangle$. Each detector responds to the total field $E_{S}\left(x_{J}\right)+$ $E_{S^{\prime}}\left(\boldsymbol{x}_{J}\right)$. Assuming the correlation of unrelated sources vanishes, the amplitude correlation $\left\langle E_{1} E_{2}^{*}\right\rangle$ between a pair of detectors 1 , 2 is

$$
\left\langle E_{1} E_{2}^{*}\right\rangle=\mathrm{e}^{-\mathrm{i} k \psi}\left[I_{\mathrm{S}} \Lambda_{\mathrm{S}}+I_{\mathrm{S}}^{\prime} \Lambda_{\mathrm{S}}^{\prime} \mathrm{e}^{-\mathrm{i} \phi_{12}^{\mathrm{tot}}}\right] .
$$

A quantum mechanical calculation gives a similar result (Mandel \& Wolf 1995) and incorporates photon bunching, an effect known for sources with intrinsic coherence (e.g. astrophysical masers). In Eq. (3) $\Lambda_{\mathrm{S}}$ and $\Lambda_{\mathrm{S}}^{\prime}$ are constants that absorb the $1 / r^{2}$ attenuation factors plus finite size effects normally of order unity (Appendix A.1). Symbol $\phi_{12}^{\text {tot }}$ is the total phase difference from propagation between source and detectors, and $\psi$ is an "overall phase" factored out for convenience.

The phases are developed using the Green function for propagation of light from source to detectors. In the frequencydomain the Helmoltz Green function $G_{x_{J}, r}$ is

$G_{x_{J}, \boldsymbol{r}}=\frac{1}{4 \pi} \frac{\mathrm{e}^{\mathrm{i} k\left|\boldsymbol{r}-\boldsymbol{x}_{J}\right|}}{\left|\boldsymbol{r}-\boldsymbol{x}_{J}\right|} \sim \frac{\mathrm{e}^{\mathrm{i} k r}}{4 \pi r} \mathrm{e}^{-\mathrm{i} k \hat{\boldsymbol{r}} \cdot \boldsymbol{x}_{J}} \exp \left(\frac{\mathrm{i} k}{2 r} \sum_{i, j=1}^{3} x_{J}^{i} \delta_{\mathrm{T}}^{i j} x_{J}^{j}\right)$.

We can drop $\mathrm{e}^{\mathrm{i} k r}$ henceforth. Change variables to the detector separation $\Delta \boldsymbol{x}_{12}$ and "detector center of mass" or average coordinate $\boldsymbol{X}_{12}$ :

$\Delta x_{12}=x_{2}-x_{1}$

$X_{12}=\left(x_{1}+x_{2}\right) / 2$.

By algebra

$$
\begin{aligned}
\phi^{\text {tot }} & =\phi_{12}^{0}+\phi_{12}^{\text {parallax }} \\
\phi_{12}^{0} & =k \Delta \boldsymbol{x}_{12} \cdot\left(\hat{\boldsymbol{r}}-\hat{\boldsymbol{r}^{\prime}}\right) \\
\phi_{12}^{\text {parallax }} & =-k\left(\frac{1}{r}-\frac{1}{r^{\prime}}\right) \Delta \boldsymbol{x}_{12} \cdot \delta_{\mathrm{T}} \cdot \boldsymbol{X}_{12} .
\end{aligned}
$$

The last line uses matrix notation. Equations (4), (5) organize the phase into a series in powers of $1 / r, 1 / r^{\prime}$. It can be extended straightforwardly to higher orders. Only one symbol $\delta_{\mathrm{T}}$ occurs in Eq. (5) because $\delta_{\mathrm{T}}(r)^{i j} \sim \delta_{\mathrm{T}}\left(r^{\prime}\right)^{i j} \sim \delta_{\mathrm{T}}$ plus negligible terms of even higher order in $1 / r$ or $1 / r^{\prime}$ relative to the order retained. To the same order of approximation the overall phase $\psi$ is

$\psi=\hat{\boldsymbol{r}} \cdot\left(\boldsymbol{x}_{1}-\boldsymbol{x}_{2}\right)-\sum_{i, j=1}^{3}\left(x_{1}^{i} x_{1}^{j}-x_{2}^{i} x_{2}^{j}\right) \delta_{\mathrm{T}}^{i j}(\boldsymbol{r}) / 2 r$.

This phase cancels under many circumstances, as we discuss below.

All higher order correlations from two sources can be expressed as products of two point amplitude correlations. The pairwise intensity-intensity correlations $\left\langle I_{1} I_{2}\right\rangle=$ $\left\langle\left|E\left(\boldsymbol{x}_{1}\right)\right|^{2}\left|E\left(\boldsymbol{x}_{2}\right)\right|^{2}\right\rangle$ is:

$$
\begin{aligned}
\left\langle I_{1} I_{2}\right\rangle= & \left(I_{\mathrm{S}} \Lambda_{\mathrm{S}}+I_{\mathrm{S}}^{\prime} \Lambda_{\mathrm{S}}^{\prime}\right)^{2}+I_{\mathrm{S}}^{2} \Lambda_{\mathrm{S}}^{2}+I_{\mathrm{S}}^{\prime 2} \Lambda_{\mathrm{S}}^{\prime 2} \\
& +2 I_{\mathrm{S}} I_{\mathrm{S}}^{\prime} \Lambda_{\mathrm{S}} \Lambda_{\mathrm{S}}^{\prime} \operatorname{Re}\left(\mathrm{e}^{\mathrm{i} \phi_{12}^{\text {tot }}}\right) .
\end{aligned}
$$

Intensity correlations can be used at both radio and optical frequencies, and have certain advantages in automatically canceling the overall phase. Higher order correlations have been proposed to improve the sensitivity in intensity correlations (Ofir \& Ribak 2006).

\subsection{Distance and relative velocity measurement}

Distance and relative velocity measurement is based on noticing that (1) correlations can be measured, (2) $\phi^{\text {parallax }}$ exists, and (3) the changes in $\phi^{\text {parallax }}$ can be extracted experimentally on the basis of translational properties. Two sources at exactly the same distance $r, r^{\prime}$ yield negligible effect. It is simplest to consider 
two sources at sufficiently different distances that one may be considered to lie at infinity. The various cases of two sources at comparable distances can also be developed when needed.

We will call the more distant object a "reference" for the source whose distance we aim to measure. The process of using a far source as a "phase reference" is standard in radio astronomy. Gwinn et al. (1986a,b) give a thorough discussion, and it is the topic of current textbooks (Thompson et al. 2001). The kind of referencing we are discussing is extremely direct, and absolutely primitive compared to the processes used in image formation.

We turn to using Eq. (5) to determine distances from the translational dependence of $\phi_{12}^{\text {parallax }}$. Both the sources and the detector pair can translate, and only the relative translation is observable. Although obvious on basic grounds, the way that translational symmetry is maintained is rather subtle. It is good to go through the exercise of finding the relative translation in two conceptually different situations:

- Consider a translation of the detectors with the sources fixed. Physically the detectors are translated across regions of slightly different curvature that are "fixed in space" by the distant sources. Then Eq. (5) gives

$$
\begin{aligned}
\boldsymbol{X}_{12} & \rightarrow \boldsymbol{X}_{12}+\Delta \boldsymbol{X}_{12} ; \\
\phi_{12}^{0} & \rightarrow \phi_{12}^{0} ; \\
\phi_{12}^{\text {parallax }} & \rightarrow \phi_{12}^{\text {parallax }}+\Delta \phi_{12}^{\text {parallax }} ; \\
\Delta \phi_{12}^{\text {parallax }} & =-k\left(\frac{1}{r}-\frac{1}{r^{\prime}}\right) \Delta \boldsymbol{x}_{12} \cdot \delta_{\mathrm{T}} \cdot \Delta \boldsymbol{X}_{12} .
\end{aligned}
$$

The correlation phase $\phi_{12}^{\text {tot }}$ therefore has a constant part, plus a term changing linearly under detector translations. Since $k \Delta \boldsymbol{x}_{12}$ are known, then extracting dependence of $\Delta \phi_{12}^{\text {tot }}$ on $\Delta \boldsymbol{X}_{12}$ yields the $\left(1 / r-1 / r^{\prime}\right)$ term to measure distances.

- Consider a translation of the sources, with the detectors fixed. Physically the local wave curvatures at the detectors change slightly from source motions in the past reaching them. Then $\hat{\boldsymbol{r}}, \hat{\boldsymbol{r}}^{\prime}$ and $\phi_{12}^{0}$ also develop corrections of order $1 / r$ or $1 / r^{\prime}$ that must be taken into account:

$$
\begin{aligned}
\boldsymbol{r}\left(\boldsymbol{r}^{\prime}\right) & \rightarrow \boldsymbol{r}\left(\boldsymbol{r}^{\prime}\right)+\Delta \boldsymbol{r} ; \\
\phi_{12}^{0} & \rightarrow \phi_{12}^{0}+k\left(\frac{1}{r}-\frac{1}{r^{\prime}}\right) \Delta \boldsymbol{x}_{12} \cdot \delta_{\mathrm{T}} \cdot \Delta \boldsymbol{r} ; \\
\phi_{12}^{\text {parallax }} & \rightarrow \phi_{12}^{\text {parallax }} .
\end{aligned}
$$

This produces the same shift in $\phi_{\text {tot }}$ as obtained from Eq. (5) if we replace $\Delta \boldsymbol{X}_{12} \rightarrow-\Delta \boldsymbol{r}$.

- Finally if both the source and the detectors are translated by the same vector then $\phi_{\text {tot }}$ is invariant due to translational symmetry.

The upshot is that physical observables depend on $\Delta \boldsymbol{X}_{12}$, which is the net translation of the detector center of mass relative to a source-reference center of mass, which is naturally independent of the coordinate origin. With this concept issue settled, the observer can fix the zero points of phases and origins in any consistent way he choses. Multiple determination of phases using more than one reference is also possible, and may be important. Its use for improving image qualities has been demonstrated in Fomalont \& Kopeikin (2002).

\subsection{Curved wave geometry}

In this subsection we give a qualitative interpretation which captures the origin of the parallax phase shift, and its effects on correlations from random sources.

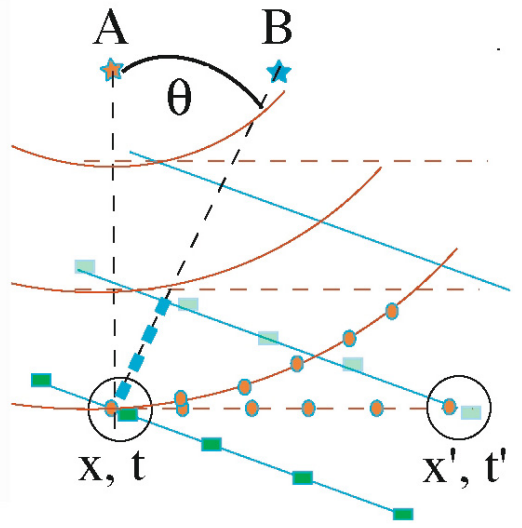

Fig. 2. Curved versus plane wave geometry. Independent fluctuations (beads or boxes) from sources $A, B$ are observed at $x, t$ and $\boldsymbol{x}^{\prime}, t^{\prime}$. The plane wave approximation (dashed wavefronts) predicts slightly different correlations compared to including the effects of wave curvature, which are described by $\phi_{12}^{\text {parallax }}$.

Consider the wave geometry of Fig. 2. A fluctuation from source $B$ is pictured as a propagating series of "boxes" measured at points $\boldsymbol{x}$, time $t$ and also at $\boldsymbol{x}^{\prime}$, time $t^{\prime}$. The fluctuations of $B$ are random and correlated with nothing but themselves. Random fluctuations from source $A$ ("dots"in Fig. 2) also occur and propagate independently. The coincidence of a $B$-fluctuation with one from $A$ depends on the orientation of the sources and detectors, the detector separations, and the source distances. In the plane wave approximation trigonometry gives "Bragg's Law" for correlations centered on $c\left(t-t^{\prime}\right) \sim\left|\boldsymbol{x}-\boldsymbol{x}^{\prime}\right| \sin \theta$ (Fig. 2). This is precisely the time-domain representation of $\phi_{12}^{0}$ and Eq. (4) in a detector with infinite bandwidth. Due to curvature of wave fronts (Fig. 2) the plane wave approximation breaks down as the translational baseline is changed. The extra phase difference taking into account curved wave propagation is of order $1 / r$, is cumulative under steady translation, and is proportional to the distance translated $\Delta \boldsymbol{X}$. The two point correlation itself scales like the detector separation $\Delta x / \lambda$, for wavelength $\lambda$. Overall the geometry of curved waves causes a phase $\phi_{12}^{\text {parallax }} \sim \Delta \boldsymbol{X} \Delta x / \lambda r$.

We then outline the conceptual steps of an interferometric parallax distance measurement:

- the observer measures the two point correlation at a particular location of the detector pair. The phase of the correlation depends on the location of the detector pair, which can be set arbitrarily;

- the observer measures the two point correlation at a translated location of the detector pair;

- the two measurements are compared. They will disagree by the effects of net translations;

- assume that the two baselines are aligned to sufficient accuracy such that the change in the leading order phase is negligible. Then the difference of correlations is proportional to $\cos \left(2 k \Delta \boldsymbol{x} \cdot \delta_{\mathrm{T}} \cdot \Delta \boldsymbol{X}\left(1 / r-1 / r^{\prime}\right)\right)$. Assuming one source at $r$ is relatively closer than the other, the distance $r$ is directly extracted.

Note that dependence of orientation of $\Delta \boldsymbol{x}$ relative to $\Delta \boldsymbol{X}$ is explicit in the formulas. It is interesting and important that the distance factor $\left(1 / r-1 / r^{\prime}\right)$ is not particular sensitive to the relative orientation of the detectors and source. A gross orientation error of one degree would only develop a relative error in the distance of order $10^{-2}$ or smaller. 
Thus the distance to the more nearby source is determined when the phase shift from parallax effects accumulates to an observable value.

\subsection{Orders of magnitude}

Here we consider order of magnitude estimates on the relative size of interferometric parallax effects.

Values to estimate $\Delta \boldsymbol{X}$ come from two causes: a uniformly translating part from the transverse projection of source proper velocity, and an annually oscillating part from the pair center $\boldsymbol{X}_{12}$ orbiting the Sun. Each term is independently observable over time as in traditional trigonometric parallax. A transverse relative velocity of order $200 \mathrm{~km} \mathrm{~s}^{-1}$ translates to about $40 \mathrm{AU} / \mathrm{year}$, while the annual oscillation of $\Delta \boldsymbol{X}$ is of order $1 \mathrm{AU}$.

Finite source size is important, and reviewed in the Appendix. Suppose the apparent angular extent of the source is $\delta \theta_{\mathrm{s}}$. The source will appear pointlike and not be resolved by a detector of effective aperture $\Delta x$ so long as $\delta \theta_{\mathrm{s}} \lesssim \Delta x / \lambda$. Let the projected transverse source size be $a=\delta \theta_{\mathrm{s}} r$. For maximal coherence with a source that appears pointlike, $k \Delta x \lessgtr r / a$, which with Eq. (5) gives $\phi_{12}^{\text {parallax }} \lesssim(X / a)\left(1-r / r^{\prime}\right)$. To order of magnitude, the parallax phase for $r \gg r^{\prime}$ is approximately the distance translated in units of the source size. Small sources are best. Fortunately QSO/AGN sources are believed to be sufficiently small that translations over scales of AU will resolve them.

Next we consider the relative size of the parallax and leading order phase. Let $\Delta \theta$ be the angular separation of a source pair. From Eq. (5) we have the leading order phase

$\phi_{12}^{0} \sim \Delta \theta /\left(\lambda / \Delta x_{12}\right)$.

Let $\Delta L$ be the typical magnitude of $\Delta \boldsymbol{X}$ (including source motion $\left.\Delta r\left(\Delta r^{\prime}\right)\right)$ over the running time of the experiment. The integrated change due to translations is

$\Delta \phi_{12}^{\text {parallax }} \sim \Delta L / r /\left(\lambda / \Delta x_{12}\right)$.

Both phases depend on $\lambda / \Delta x_{12}$, the typical angular resolution from optics. The parallax phase change is of order one if the transverse baseline of translation $\delta_{\mathrm{T}} \cdot \Delta L$ can be resolved by an instrument of aperture $\Delta x$ observing from distance $r$. Since $\Delta L \gg \Delta x$ is always possible, the parallax resolution is dominated by the translation scale, not the apparent correlation aperture of the instrument.

Numerical evaluation gives

$$
\begin{gathered}
\phi_{12}^{0} \sim 10^{4} \mathrm{rad}\left(\frac{\Delta \theta}{\operatorname{arcsec}}\right)\left(\frac{1 \mathrm{~cm}}{\lambda}\right)\left(\frac{\Delta x_{12}}{10^{4} \mathrm{~km}}\right) ; \\
\phi_{12}^{\text {parallax }} \sim 10^{-1} \operatorname{rad}\left(\frac{\Delta \boldsymbol{X}}{\mathrm{AU}}\right)\left(\frac{100 \mathrm{kpc}}{r}\right)\left(\frac{1 \mathrm{~cm}}{\lambda}\right)\left(\frac{\Delta x_{12}}{10^{4} \mathrm{~km}}\right) .
\end{gathered}
$$

Let $\delta \phi_{\text {err }}$ represent the typical error in observed phases. To order of magnitude the parallax phase can be measured when $\phi_{12}^{\text {parallax }} \gtrsim \delta \phi_{\text {err }}$. Expressing $\delta \phi_{\text {err }}$ in units of degrees, the order of magnitude of the maximum distance observable $r_{\text {max }}$ yields

$r_{\max } \sim 1 \mathrm{Mpc}\left(\frac{1^{\circ}}{\delta \phi_{\mathrm{err}}}\right)\left(\frac{\Delta L}{\mathrm{AU}}\right)\left(\frac{\Delta x}{10^{4} \mathrm{~km}}\right)\left(\frac{1 \mathrm{~cm}}{\lambda}\right)$.

Concrete estimates of $\delta \phi_{\text {err }}$ depend on many factors of instrument calibration and running time. Using picosecond overall resolution at $10 \mathrm{Ghz}$ frequencies and $\Delta L \sim 1 \mathrm{AU}$ gives $r_{\max } \sim 1 \mathrm{Mpc}$. Optical wavelengths $10^{5}$ times smaller lead to a correspondingly larger estimate of $r_{\max }$, suggesting it may be possible in principle to measure distances to the observable edge of the Universe.

The relative size of the leading order phase $\phi_{12}^{0}$ is a serious complication. One means to control uses a source-separation $\Delta \theta$ that is as small as possible. The usual image-making restriction that two sources be resolved at different positions of the baseline is absolutely not necessary to maintain. The leading order phase cancels in the 4-point correlations, as we discuss next.

\subsection{4-point correlations}

In this section we consider the situation where rapid variations of the leading phase might tend to wash out the more slowly varying parallax phase. To control the effects of $\phi^{0}$ we can (in effect) measure it twice, using another pair of detectors $(3,4)$, separated by nearly the same offset:

$\Delta \boldsymbol{x}_{34}=\Delta \boldsymbol{x}_{12}+\boldsymbol{\eta}$.

It is clear that $|\boldsymbol{\eta}| \ll\left|\Delta \boldsymbol{x}_{12}\right|$ can be made relatively small with great precision.

Consider the product of $\left\langle I_{1} I_{2}\right\rangle\left\langle I_{3} I_{4}\right\rangle$, which is one of the terms in the 4-point intensity correlation. Denoting the overall normalizations by $N_{1}$ and $N_{2}$ we write

$\left\langle I_{1} I_{2}\right\rangle=N_{1}+N_{2} \cos (A+B)$

where $A=\phi_{12}^{0}, B=\phi_{12}^{\text {parallax }}$. Express $A=\bar{A}+\delta A$, where $\bar{A}$ and $\delta A$ denote a constant and a fluctuating or variable component, respectively. Due to the large typical size of $A$ compared to $B$, we must assume that $\delta A \gg B$ causes a problem and might wipe out the signal. Isolate rapidly varying factors, writing

$\left\langle I_{1} I_{2}\right\rangle=N_{1}+N_{2}[\cos (\bar{A}+B) \cos \delta A-\sin (\bar{A}+B) \sin \delta A]$.

Write a similar relation for $\left\langle I_{3} I_{4}\right\rangle=N_{1}^{\prime}+N_{2}^{\prime} \cos \left(A^{\prime}+B^{\prime}\right)$, where $A^{\prime}=\bar{A}^{\prime}+\delta A^{\prime}$. Then $\left\langle I_{1} I_{2}\right\rangle\left\langle I_{3} I_{4}\right\rangle$ contains 9 terms, which consist of constants, terms linear in a rapidly varying cosines or sines, and terms bilinear.

The detection strategy allows the rapid variations to occur, while averaging over $K \gg 1$ cycles of $\cos \delta A \ldots . \cos \delta A^{\prime}$. Let $\langle\langle\rangle\rangle$ denote this average. As clear from Sect. 2.4, the value of $K$ may be millions or more while accumulating a single oscillation of $\phi^{\text {parallax }}$. Then products with an odd number of rapid cosines or sines can be dropped with a relative error of order $1 / K$. The remaining term with two cosines gives

$$
\begin{aligned}
\left\langle\left\langle\cos (A+B) \cos \left(A^{\prime}+B^{\prime}\right)\right\rangle\right\rangle= & \left\langle\left\langle\cos A \cos A^{\prime}\right\rangle\right\rangle \cos B \cos B^{\prime} \\
& +\left\langle\left\langle\sin A \sin A^{\prime}\right\rangle\right\rangle \sin B \sin B^{\prime}
\end{aligned}
$$

We may arrange the geometry such that $A \approx A^{\prime}$ to order one over the averaging period. Then have

$\left\langle\left\langle\cos A \cos A^{\prime}\right\rangle\right\rangle=\left\langle\left\langle\sin A \sin A^{\prime}\right\rangle\right\rangle=1 / 2$.

The calculation receives a non-zero contribution from those regimes when the rapidly varying terms coincide. The final result is

$$
\begin{aligned}
\left\langle\left\langle\left\langle I_{1} I_{2}\right\rangle\left\langle I_{3} I_{4}\right\rangle\right\rangle\right\rangle & =N_{1} N_{1}^{\prime}+N_{2} N_{2}^{\prime} \cos \left(B-B^{\prime}\right) \\
& =N_{1} N_{1}^{\prime}+N_{2} N_{2}^{\prime} \cos \left(\phi_{12}^{\text {parallax }}-\phi_{34}^{\text {parallax }}\right) .
\end{aligned}
$$

Thus with a 4-point correlation the parallax phase is isolated from the rapidly varying background.

Note that the net translation of the 3-4 receiver pair is generally independent of the 1-2 pair. Then it is straightforward to 
arrange for the surviving slow oscillation to produce a net signal. A simple configuration (Fig. 1) puts $\Delta \boldsymbol{x}_{12}=\Delta \boldsymbol{x}_{34}=\Delta \boldsymbol{x}, \boldsymbol{x}_{3}=$ $-\Delta \boldsymbol{X}-\Delta \boldsymbol{x} / 2, \boldsymbol{x}_{4}=-\Delta \boldsymbol{X}+\Delta \boldsymbol{x} / 2$. The difference term $\boldsymbol{\eta}$ is relatively negligible and was dropped. The parallax terms add, producing an oscillation going like $\cos \left(2 k \Delta \boldsymbol{x} \cdot \delta_{\mathrm{T}} \cdot \Delta \boldsymbol{X}\left(1 / r-1 / r^{\prime}\right)\right)$. Again the parallax phase is isolated from a rapidly varying background.

There may be advantages to directly correlating the signal from four receivers, either at the amplitude or the intensity level. Let $\left\langle I_{1} I_{2} I_{3} I_{4}\right\rangle$ be the raw four-point intensity correlation. It is the product of $4^{4}$ terms, many of which average to zero or are similar to those terms evaluated for the two-point intensity correlation. We spare the reader extensive algebra. Dropping terms that oscillate rapidly, and with $\boldsymbol{\eta} \rightarrow 0$, a direct calculation gives

$$
\left\langle I_{1} I_{2} I_{3} I_{4}\right\rangle=\mathcal{N}_{1}+\mathcal{N}_{2} \cos \left(\phi_{12}^{\text {parallax }}-\phi_{34}^{\text {parallax }}\right) .
$$

Here $\mathcal{N}_{1}$ and $\mathcal{N}_{2}$ are normalizations depending on the intensity of the two sources. The other terms in the four point correlation either vanish after statistical averaging or reduce to the two terms given in Eq. (11). The remarkable cancellations in the 4-point intensity correlation show that the parallax phase can be measured in terms of a standard statistical description of raw data.

Similarly, the 4-point amplitude correlation (observable at radio frequencies) consists of sums of terms with phases $\phi^{0}$ and $\phi^{\text {parallax }}$, along with "sum-phases" of the form $\left(\boldsymbol{k}_{1}+\boldsymbol{k}_{2}\right)$. $\Delta \boldsymbol{x}$. Sum-phases in conventional long-baseline interferometry (Mandel \& Wolf 1995; Scully \& Zubairy 1997; Thompson et al. 2001) tend to cause difficulty due to atmospheric fluctuation effects. Intensity interferometry is much less sensitive, as positioning accuracy is set by the coherence time and not the wavelength (LeBohec \& Holder 2005).

Continuing with higher order correlations, the prospects of multiple-detection (overdetermination) of phases is absolutely relevant. Multiple determination in radio synthesis (say) is already a highly-developed art, which simply need the parallax phase effects to be incorporated.

\subsection{Comparison with trigonometric parallax}

Trigonometric parallax of 61 Cygni was first demonstrated by Bessel in 1838. Its basic principles remain unchanged to this day. The analysis of trigonometric parallax is elementary yet interesting to compare to interferometric parallax.

Consider a single point source at distance $r_{*}$ at an angle $\theta_{*}$. The star's angular position is first measured by making an image with an angular resolution of order $\Delta \theta_{\text {res }} \sim \lambda / D$, where $\lambda$ is the wavelength and $D$ is the aperture. Under a translation by $\Delta \boldsymbol{X}$ of the telescope relative to the star, the angle changes by ${ }^{2} \delta \theta_{*}$, of order

$\delta \theta_{*} \sim \Delta \boldsymbol{X} / r_{*}$.

An unambiguous measurement of parallax requires that the apparent shift $\delta \theta_{*}$ be larger than the resolution, giving

$$
\begin{aligned}
\frac{\Delta \boldsymbol{X}}{r_{*}} & \approx \frac{\lambda}{D} ; \\
\frac{\Delta \boldsymbol{X} D}{\lambda r_{*}} & \approx 1 .
\end{aligned}
$$

With modern instruments performance better than the Rayleigh resolution criteria can be achieved. The position of a peak in an

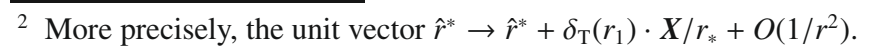

image map can be fit to better accuracy than the naive resolution. The errors of fitting depend on the signal to noise. By combining a number of techniques, very impressive parallax measurements to kpc distances have been demonstrated with VLBI radio telescope arrays (Felli \& Spencer 1989; Hachisuka et al. 2006). All of these methods depend on generating a very high quality image with precisely known systematic errors.

What differs between trigonometric and interferometric parallax? There are several points that depend on the realization.

- Interferometry is nearly unique in ability to measure small effects. While the extensive signal processing to go from thousands to millions of separate phases to images contains one generalized form of "phase information", it is clearly advantageous to isolate those particular and special phase shifts uniquely associated with distance and transverse velocity measurements;

- with interferometric parallax it is not necessary to make a high precision measurement of the angular position of the source, nor even necessary to image the source at all;

- a host of "pointing" variables associated with precisely locating the position of an object might be entirely sidestepped by parallax phase measurements;

- high precision of baseline alignments is nevertheless needed in interferometric parallax. In some sense this is the analog of precise angular position and image quality measurement in trigonometric parallax. Note that modern astrometry can achieve stunning precision of alignments in the solar system - errors comparable to a few meters over an astronomical unit;

- in some special cases the leading phase $\phi^{0}$ may be very small, so that the requirement of baseline alignment is not very stringent. This may happen if the two sources under consideration have very small angular separation;

- by using phase information more directly and efficiently, one should be able to make better direct distance measurements in any given running time than when statistical weight is invested in constructing images. We have investigated questions of signal/noise, and consulted with experts (Mutel 2007) in an attempt to quantify what might be achievable. In the end signal/noise estimates depend strongly on experimental "reality" factors which require intimate information about detector performance best left for specialized studies;

- complications can be minimized when one is extracting a small amount of information rather than a detailed image. The complicating role of fluctuations of the "sum phase" in amplitude correlations is well known (Thompson et al. 2001). Meanwhile intensity correlations self-cancel the overall phase, while directly yielding the difference and parallax phase. Without making any images, Hanbury-Brown \& Twiss $(1954,1956)$ were able to measure intensity correlations on $100 \mathrm{~m}$ length scales with an Earth-bound detector using crude photodetectors more than 4 decades ago. Coherent (Michelson) interferometry on such a scale was impossible at the time, if only because of atmospheric fluctuations. Many of the precision instrumental requirements of interferometry are ameliorated with intensity correlations. Indeed intensity correlations at optical frequencies can be developed by counting photons that enter widely separated detectors. This suggests that intensity correlations may find new applications to distance measurements. 


\section{Conceptual applications}

While most of our analysis is necessarily conceptual, we examined a few specific cases in order to assess the feasibility of distance and velocity measurements using interferometric parallax.

\subsection{Specific examples}

\subsubsection{Water masers: a galactic pilot experiment at radio frequencies}

Consider measurement of a water maser source in our galaxy referenced by a distant AGN. Hachisuka et al. (2006) report parallax measurements of the star-forming region $\mathrm{W} 3(\mathrm{OH})$ using VLBA measurements of unprecedented accuracy. The measured annual parallax is $0.489 \pm 0.017$ milli-arcsec (mas), corresponding to a distance of $2.04 \pm 0.07 \mathrm{kpc}$. Hachisuka et al. (2006) made 7 measurements of $4 \mathrm{~h}$ duration spaced over the period of about a year. After making image maps and using JMFIT to fit multiple power peaks the position errors of each measurement on the best source is about 0.1 mas (Fig. 5 in Hachisuka et al. 2006). With the global fit to the annual periodicity, errors in the overall measurement drop to the values reported of order $10 \mu$ as. Maser source with high power and stability, plus sufficient running time to achieve good signal to noise, is a key to achieving such high resolution.

Using frequency $22.5 \mathrm{Ghz}, \Delta \boldsymbol{X}=1 \mathrm{AU}$, and $\delta x=10^{4} \mathrm{~km}$, we estimate the parallax phase change $\phi^{\text {parallax }} \sim 5$ rad. Over time the rate of accumulation is $\mathrm{d} \phi^{\text {parallax }} / \mathrm{d} t \sim 5 \%$ day. Since the error $\delta r / r \sim \delta \phi^{\text {parallax }}$ one would estimate that a $2 \%$ relative error in $r$, comparable to Hachisuka et al. (2006), might be achieved with an error $\phi^{\text {parallax }} \sim 1^{\circ}$. This is perhaps naive, yet reasonably consistent with the Hachisuka et al. (2006) measurement.

We believe it would be interesting to perform a pilot experiment measuring $\phi^{\text {parallax }}$ and bypassing the steps of image reduction. Note that the accuracy obtained from fitting image maps is inherently limited by signal/noise. The extraction of $\phi_{12}^{\text {parallax }}$ should be quite robust even integrating over conditions of varying source intensity. More efficient use of phase information without the intermediate complications of maps might credibly reduce the need for long running times. Long running times are not only impractical use of facilities, but also impossible with the high time variability of $\mathrm{H}_{2} \mathrm{O}$ masers whose features often change over a one-year time scale. Perhaps intensity correlations at radio frequencies, which have but one phase to measure, and no overburden of overall phase to account for, might also be particularly efficient. It is interesting in this regard that the dominant errors cited in Hachisuka et al. (2006) are due to atmospheric corrections to which intensity correlations are rather insensitive.

\subsubsection{Optical interferometric pilot experiment}

SIM is an ambitious space-borne optical interferometer with launch planned in 2015. As with radio parallax the thrust of SIM for distance measurements lies in first establishing highly precise angular positions and then tracking changes over time. SIM is basically an optical Michelson interferometer with aperture $\Delta x \sim 9 \mathrm{~m}$ and operating in the wavelength range of $\lambda \sim$ $0.4-0.9 \mu \mathrm{m}$.

An instrument with the size and wavelength parameters of SIM should develop $\phi^{\text {parallax }} \sim 0.02 \operatorname{rad}(1 \mathrm{kpc} / r)$ for $\Delta \boldsymbol{X}=$ 1 AU. If the source and its reference are chosen to be separated by order arc-sec, the fluctuations in the leading phase $\phi^{0}$ due to baseline angular orientation errors are comparable to the parallax phase for distance of order $1 \mathrm{Mpc}$. Hence measurement of distances of order Mpc may be possible using interferometric parallax with SIM. If we arbitrarily degrade this estimate by a factor of 1000, measurements at kpc distances remain extremely interesting. Measurement may also be facilitated by using an optimal alignment discussed in the Appendix.

Most impressively one need not make the estimated $\$ 500$ million investment of SIM to run a test. Intensity correlations on the $10-\mathrm{m}$ distance scale and using recycled searchlight mirrors were demonstrated about 50 years ago by Hanbury-Brown \& Twiss $(1954,1956)$, yet without ever referring to the parallax phase. We suggest that a reasonable distancemeasuring pilot experiment might be conducted with cosmic ray facilities such as the HESS or WHIPPLE experiments (LeBohec \& Holder 2005).

\subsubsection{Gigaparsec ambitions}

The ultimate ambition for distance measurement would reach to the Gpc scale of interest in Big Bang cosmology. For Gpc distances, one needs to measure a relatively small phase shift or push the limits of baselines to much larger than $10^{4} \mathrm{~km}$ or extend wavelength to the sub-mm range. Such ambitions would require a dedicated observatory, but still appear to be within the reach of current day technology.

As previously mentioned, quasar sources are believed to have physical sizes extending to the range of $1 \mathrm{AU}$, whereby $\delta \theta_{\mathrm{s}} \sim 10^{-9}$ arcsec. Black hole, supernova and GRB sources are of course even smaller than QSO's, with correspondingly larger coherence zones. It may be possible to find another source at a comparable distance within an acceptable angular separation. It might also be possible to measure gravitationally lensed single objects, exploiting two path lengths $r, r^{\prime}$. Admittedly such coincidences are rare, but a single direct measurement of the distance to a QSO at Gpc distance would be revolutionary. If sources satisfy finite size criteria, there remains the need to maintain high detector orientation accuracy.

The scale of accuracy needed depends on the detector separation $\triangle x$. The joint Japanese/US VSOP (VLBI Space Observatory Program) mission had a $21000 \mathrm{~km}$ orbit and an $8 \mathrm{~m}$ telescope (Horiuchi et al. 2004). The Russian program RADIOASTRON proposes very large orbits (see the website, www.asc.rssi.ru/radioastron/). It is at least possible to conceive $\Delta x \sim \mathrm{AU}$ with a collection of satellites in solar orbit. Establishing correlations retrospectively between widely separated detectors requires exacting knowledge of orientation and relative position errors. Just how demanding are the requirements?

Note that $\phi^{0}$ is translationally invariant and stands as an irrelevant constant when extracting $\phi_{12}^{\text {parallax }}$ under translations. The variation due to experimental error on $\phi^{0}$ is important. The most stringent requirement is imposed by the need to accurately align baselines. With a source separation $\Delta \theta \sim 1$ arcsec and $r \sim \mathrm{Gpc}$ the ratio $\phi^{0} / \Delta \phi^{\text {parallax }} \sim 10^{9}$. If $\Delta \phi^{\text {parallax }} \sim 10^{-3}$ can be measured an error $\delta \phi^{0} / \phi^{0} \lesssim 10^{-6}$ is tolerable. This in turn requires angular orientation errors of $\boldsymbol{\Delta} x, \boldsymbol{\Delta} \boldsymbol{X} \lesssim 10^{-6}$. The angular orientation errors reported for SIM already lie below this level. At the time of the review of Sovers et al. (1998), Earth-based VLBI had already established an absolute inertial frame with sub-milliarcsecond accuracy. In terms of distances an error $\delta x \sim 100 \mathrm{~m}$ is tolerable for $\Delta x \sim 1$ AU separation baselines. Proven satellite ranging techniques (Thornton \& Border 2003) have achieved accuracies superior by orders of magnitude. The optimal alignment 
technique discussed in the Appendix offers further advantages that cancel first order angular errors.

Therefore, to the extent that we can foresee barriers of principle, we cannot find a reason that a determined effort by the present or future civilization could not directly measure distances on the entire scale of the Universe.

\subsubsection{Photon counting estimates}

There appears to be a prejudice in present day astronomy that intensity correlations are disfavored due to "low signal/noise." Statements to this effect appear in textbooks (Thompson et al. 2001) without much elaboration. Consulting Eq. (6), intensity correlations have a constant background of the same order as the oscillating signal, which is somewhat less favorable than a purely oscillating signal with zero background. Yet such details are small effects compared to wide variations of different terms in our study, suggesting optical intensity correlations deserve a fresh re-examination.

The signal/noise $S / N$ of intensity correlation measurements (Hanbury-Brown \& Twiss 1956; LeBohec \& Holder 2005) scales like

$$
\begin{aligned}
S / N & \sim \alpha A \gamma_{\mathrm{d}}^{2} \frac{\mathrm{d} N_{\gamma}}{\mathrm{d} A \mathrm{~d} t \mathrm{~d} f} \sqrt{\Delta f T / 2}, \\
& \sim \frac{\alpha A \gamma_{\mathrm{d}}^{2}}{\sqrt{2}} \sqrt{\frac{T}{\Delta f}} \int_{f_{0}}^{f_{0}+\Delta f} \frac{\mathrm{d} N_{\gamma}}{\mathrm{d} A \mathrm{~d} t \mathrm{~d} f} .
\end{aligned}
$$

Here $A$ is the detector area, $\gamma_{\mathrm{d}}^{2}$ the degree of coherence, $\alpha$ the quantum efficiency, $\Delta f$ is the bandwidth of the correlation, and $T$ the running time on source. The number of photons per area per frequency is $\mathrm{d} N_{\gamma} / \mathrm{d} A \mathrm{~d} t \mathrm{~d} f$. The second line in Eq. (12) shows that decreasing the bandwidth increases $S / N$, which was exploited by Hanbury-Brown \& Twiss $(1954,1956)$ using simple optical filters. Using Eq. (12) is more specific and considerably more conservative than the photon-counting phase error $\delta \phi / \phi \sim 1 / \sqrt{n}$ for $n$ photons that is commonly used in astrometry (Lindegren 1978, 2005).

For the photon flux we rescaled the calculations of Ulmer et al. (2004). We assumed a $100 \mathrm{~m}^{2}$ aperture with $10 \%$ throughput and detection efficiency, and Hubble constant $H=0.7 \times$ $100 \mathrm{~km} \mathrm{~s}^{-1} \mathrm{Mpc}^{-1}$. There are 20 photons per second in $\mathrm{H}_{\alpha}$ light for a typical bright galaxy with power $10^{42} \mathrm{ergs} / \mathrm{s}$ at redshift $z \sim 1$. This determines $A(\mathrm{~d} N / \mathrm{d} A \mathrm{~d} t \mathrm{~d} f) \Delta f$. Using $\Delta \lambda \sim 1 A^{0}$ for efficient $\mathrm{H}_{\alpha}$ filters, and point-like sources with $\gamma_{\mathrm{d}}^{2} \sim 1$, then $S / N \lesssim 10^{-4} \sqrt{T / \mathrm{s}}$. The relative error in a phase $\Delta \phi / \phi \sim N / S \sim$ $10^{4} / \sqrt{T / \mathrm{s}}$. Again the leading order phase must be cancelled, either with the 4-point scheme or some other mechanism, to make such an ambitious measurement. If we conservatively choose sources separated by $\Delta \theta \sim \operatorname{arcsec}$, take $\Delta x \sim 10^{4} \mathrm{~km}$, and $\lambda \sim 6 \times 10^{-4} \mathrm{~mm}$, then $\phi^{\text {parallax }} \sim 1$. Then measurement with $\Delta \phi_{\text {parallax }} / \phi_{\text {parallax }} \sim 1$ needs $T \sim 10^{8} \mathrm{~s} \sim 3$ years on this basis. (Using $\delta \phi / \phi \sim 1 / \sqrt{n}$ would give $T \gtrsim 10^{6} \mathrm{~s}$, about one day.) The number of photons detectable increases for smaller $r$ somewhat faster than $1 / r^{2}$. A similar source at $100 \mathrm{Mpc}$ range has about 100 times the flux, decreasing the time on source $T \rightarrow 0.3$ years.

Finer estimates can undoubtedly be made given intimate knowledge of detector position errors and phase measurement technology beyond our expertise. It is also interesting to increase signal/noise by developing the highest possible collection area. There are obvious limits to collection areas of single channels. Perhaps modern signal- processing techniques might make correlations from a large number of modest-area detectors more effective than the traditional practice of using a small number of large-area detectors.

\section{Conclusion}

We have introduced a new technique for direct measurement of astronomical distances and proper motions. The technique is based on detecting wavefront curvature using interferometry. The technique may provide optimal utilization of observation time by focusing data-taking on the particular phase needed for distance measurements. Numerous topics in conventional astronomy would benefit enormously from direct measurements of distances of order kpc to Mpc. From near Earth-orbit, or perhaps with detectors fixed on Earth, it should be possible to measure distances to $r \geq \mathrm{Mpc}$, which would be magnificent. A number of radio telescopes in orbit can make mutual correlations, and correlations with ground-based receivers, to develop distance resolution well beyond any we have seen published. There is every reason to believe that a dedicated project might in principle measure Gpc distances using interferometric parallax. While there are a host of challenging technical issues for measurement of such large distances, there is every reason to believe that it should be feasible with current technology.

Acknowledgements. P.J. thanks Vasant Kulkarni, Rajaram Nityananda and Anantha Ramakrishna for useful discussions. J.P. thanks Steve Myers informing us of VSOP and RADIOASTRON, Robert Mutel for helpful discussions, and Sergei Kopeikin for useful discussions and references. Research supported in part under DOE Grant Number DE-FG02-04ER14308.

\section{Appendix A: Finite source size and optimal alignment effects}

In this Appendix we add information on finite source effects. Extending the analysis towards imaging a finite source is omitted, as it is a standard application of the plane-wave VCZ theorem. We also discuss a technique of optimal alignments.

\section{A.1. Finite source size effects}

The basic two point amplitude correlation for a single incoherent source is given by

$$
\begin{aligned}
\left\langle E\left(\boldsymbol{x}_{1}\right) E^{*}\left(\boldsymbol{x}_{2}\right)\right\rangle= & \int \mathrm{d}^{2} y\left\langle E_{\mathrm{S}}(\boldsymbol{y}) E_{\mathrm{S}}^{*}(\boldsymbol{y})\right\rangle \\
& \times \frac{\mathrm{e}^{\mathrm{i} k\left[\left|\boldsymbol{r}_{1}+\boldsymbol{y}\right|-\left|\boldsymbol{r}_{2}+\boldsymbol{y}\right|\right]}}{\left|\boldsymbol{r}_{1}+\boldsymbol{y} \| \boldsymbol{r}_{2}+\boldsymbol{y}\right|} .
\end{aligned}
$$

The argument of the exponential integrand is expanded as

$$
\begin{aligned}
\left|\boldsymbol{r}_{1}+\boldsymbol{y}\right|-\left|\boldsymbol{r}_{2}+\boldsymbol{y}\right|= & r_{1}-r_{2}+\hat{r}_{1} \cdot \boldsymbol{y}-\hat{r}_{2} \cdot \boldsymbol{y} \\
& -\left(\boldsymbol{y} \cdot \hat{r}_{1}\right)^{2} / 2 r+\left(\boldsymbol{y} \cdot \hat{r}_{2}\right)^{2} / 2 r .
\end{aligned}
$$

By inspection the last two terms give negligible contribution. Let $a$ be the projected transverse size of the "source", as measured by those Fourier components having significant contributions to the integral. A QSO, for example, may have an effective source size of order AU, while being associated with much larger extended structure. All terms involved in Eq. (A.2) are functions of source angular size $\delta \theta_{\mathrm{s}} \sim a / r$. The integrand will oscillate rapidly if $a / r \gg 1 /(k \Delta x)$, leading to a correlation that is exponentially small for typical source distribution. Conversely, as long as $|\Delta \boldsymbol{x}| \lesssim a /(k r)$ the corrections due to finite size can be absorbed into the overall factors $I_{S} \Lambda_{S}, I_{S}^{\prime} \Lambda_{S}^{\prime}$ etc. in Eq. (3), and 
a source appears to be a point. This reproduces the usual planar source criterion (Mandel \& Wolf 1995; Scully \& Zubairy 1997). It is perhaps more direct to use the Rayleigh criterion that $\delta \theta_{\mathrm{s}} \gtrsim \Delta x / \lambda$ is the smallest angular structure an aperture of order $\Delta x$ can resolve.

\section{A.2. Optimal alignments}

The order of magnitude estimates above made no special assumptions of the alignment of the detectors relative to the sources. However the alignment properties can be used to advantage.

Observe that the leading phase depends on the angular orientation by a factor of $\cos (\eta)=\Delta x_{12} \cdot\left(\hat{\boldsymbol{r}}-\hat{\boldsymbol{r}^{\prime}}\right) \rightarrow$ It is maximized when the detector pair separation is parallel to the source separation projected onto the sky. Meanwhile the observable parallax phase is proportional to $\Delta x_{12} \cdot \delta_{\mathrm{T}} \cdot \Delta \boldsymbol{X}$. The matrix $\delta_{\mathrm{T}}$ projects away vector components proportional to $\hat{\boldsymbol{r}}$, otherwise does nothing. In particular the parallax phase depends on orientation of $\Delta x_{12}$ relative to $\Delta \boldsymbol{X}$, not relative to the sources.

It is therefore possible to measure the parallax phase under conditions that the leading order phase remains relatively constant. Let $\eta \rightarrow \eta+\delta \eta$ change by a small error $\delta \eta \ll 1$, and take data near $\eta \sim 0$. Then $\cos (\eta) \sim 1-\delta \eta^{2}$ changes only by terms of order $\delta \eta^{2}$. The second order dependence makes a substantial suppression of order $10^{-4}$ for (say) $\delta \eta \sim 10^{-2}$.

The degree to which detector orientation can be manipulated to maintain $\eta \sim 0$ depends on the detector configuration, and is clearly different for Earth-bound versus space-borne arrays. We update the estimates above using units of degrees:

$$
\begin{aligned}
& \phi_{12}^{0} \sim 1 \mathrm{rad}\left(\frac{\delta \eta}{1^{\circ}}\right)^{2}\left(\frac{\Delta \theta}{\operatorname{arcsec}}\right)\left(\frac{1 \mathrm{~cm}}{\lambda}\right)\left(\frac{\Delta x_{12}}{10^{4} \mathrm{~km}}\right) \\
& \phi_{12}^{\text {parallax }} \sim 10^{-1} \mathrm{rad}\left(\frac{\Delta \boldsymbol{X}}{\mathrm{AU}}\right)\left(\frac{100 \mathrm{kpc}}{r}\right)\left(\frac{1 \mathrm{~cm}}{\lambda}\right)\left(\frac{\Delta x_{12}}{10^{4} \mathrm{~km}}\right) .
\end{aligned}
$$

\section{References}

Bartel, N. 1988, Proc. of the 129th IAU Symp., Cambridge, MA, May 10-15, 1987 (A89-13726 03-90) (Dordrecht: Kluwer Academic Publishers), 175
Bradshaw, C. F., Fomalont, E. B., \& Geldzahler, B. J. 1999, ApJ, 512, L121 Brisken, W. F., Benson, J. M., Goss, W. M., \& Thorsett, S. E. 2002, ApJ, 571, 906

Campbell, R. M., Bartel, N., Shapiro, I. I., et al. 1996, ApJ, 461, L95

Dodson, R., Legge, D., Reynolds, J. E., \& McCulloch, P. M. 2003, ApJ, 596, 1137

Felli, M., \& Spencer, R. E. 1989, Very Long Baseline Interferometry, Techniques and Applications (Dordrecht: Kluwer Academic Publishers)

Fomalont, E. B., \& Kopeikin, S. 2002, in 6th European VLBI Network Symposium on New Developments in VLBI Science and Technology, ed. E. Ros, R. W. Porcas, A. P. Lobanov, \& J. A. Zensus (Bonn: Max-Planck-Institut fuer Radioastronomie), 53

Gwinn, C. R., Taylor, J. H., Weisberg, J. M., \& Rawley, L. A. 1986a, AJ, 91, 338

Gwinn, C. R., Taylor, J. H., Weisberg, J. M., \& Rawley, L. A. 1986b, AJ, 91, 974 (erratum)

Gupta, Y., \& Dwarakanath, K. S. 1999, Low Frequency Radio Astronomy, Lecture notes, NCRA, Pune

Hachisuka, K., Brunthaler, A., Menten, K. M., et al. 2006, ApJ, 645, 337

Hanbury-Brown, H., \& Twiss, R. Q. 1954, Phil. Mag., 45, 663

Hanbury-Brown, H., \& Twiss, R. Q. 1956, Nature, 178, 1046

Honma, M., Oyama, T., Hachisuka, K., et al. 2000, Pub. Astro. Soc. Japan, 52, 631

Horiuchi, S., Fomalont, E. B., Taylor, W. K., et al. 2004, ApJ, 616, 110

Kopeikin, S., \& Schäfer, G. 1999, Phys. Rev. D, 60, 124002

Kurayama, T., Sasao, T., \& Kobayashi, H. 2005, ApJ, 627, L49

LeBohec, S., \& Holder, J. 2005, Proc. of 29th International Cosmic Ray Conf. (ICRC 2005), Pune, India [arXiv: astro-ph/0507010]

Lindegren, L. 1978, in Modern Astrometry, ed. F. V. Prochazka, R. H. Tucker, Univ. Obs. Vienna, IAU Coll., 48, 197

Lindegren, L. 2005, in GAIA 2004 Proc., 29

Mandel, L., \& Wolf, E. 1995, Optical Coherence and Quantum Optics (Cambridge: Cambridge University Press)

Mutel, R. 2007, private communication

Samuel, J. 2004, Class. Quant. Grav, 21, L83

Scully, M. O., \& Zubairy, M. S. 1997, Quantum Optics (Cambridge: Cambridge University Press)

Sjouwerman, L. O., Messineo, M., \& Habing, H. J. 2004, Pub. Astro. Soc. Japan, 56,45

Sovers, O. J., Fanselow, J. L., \& Jacobs, C. S. 1998, Rev. Mod. Phys., 70, 1393

Thompson, A. R., Moran, J. M., \& Swenson Jr, G. W. 2001, Interferometry and Synthesis in Radio Astronomy, 2nd edn. (Wiley)

Thornton, C. L., \& Border, J. S. 2003, Radiometric tracking techniques for deepspace navigation, Deep-space communications and navigation series 00-11, Jet Propulsion Laboratory

Ulmer, M. P., Kibblewhite, E. J., Herter, T. L., et al. 2004, Proc. SPIE, 5382, 193 van Langevelde, H. J., Diamond, P. J., Vlemmings, W., et al. 1999, New Astron. Rev., 43, 575

Vlemmings, W. H. T., van Langevelde, H. J., Diamond, P. J., Habing, H. J., \& Schilizzi, R. T. 2003, A\&A, 407, 213

Vlemmings, W. H. T., Chatterjee, S., Brisken, W. F., et al. 2005, Mem. Soc. Astron. It., 76, 531 